\title{
Coefficient Estimates of Certain Subclasses of Bi-Bazilevic Functions Associated with Chebyshev Polynomials and Mittag-Leffler Function
}

\author{
Adeniyi Musibau Gbolagade ${ }^{1,{ }^{*}}$ and Ibrahim Tunji Awolere ${ }^{2}$ \\ ${ }^{1}$ Department of Mathematics, Emmanuel Alayande College of Education, \\ P. M. B. 1010, Oyo, Oyo State, Nigeria \\ e-mail: gbolagadeam@yahoo.com \\ ${ }^{2}$ Department of Mathematics, Olusegun Agagu University of Science and Technology, \\ P. M. B. 353, Okitipupa, Ondo State, Nigeria \\ e-mail: awolereibrahim01@gmail.com
}

\begin{abstract}
In this present investigation, the authors introduced certain subclasses of the function class $T_{\theta}^{\alpha}(\lambda, \beta, t)$ of bi-Bazilevic univalent functions defined in the open unit disk $U$, which are associated with Chebyshev polynomials and Mittag-Leffler function. We establish the Taylor Maclaurin coefficients $\left|a_{2}\right|,\left|a_{3}\right|$ and $\left|a_{4}\right|$ for functions in the new subclass introduced and the Fekete-Szego problem is solved.
\end{abstract}

\section{Introduction}

Let $A$ denote the class of analytic function $f(z)$ in the open unit disk $U=$ $\{z:|z|<1\}$ and $H$ denote the subclass of $A$ which are normalized by the condition $f(0)=0$ and $f^{\prime}(0)-1=0$ with the representation

$$
f(z)=z+\sum_{k=2}^{\infty} a_{k} z^{k} .
$$

Received: September 19, 2020; Revised \& Accepted: October 16, 2020

2020 Mathematics Subject Classification: Primary 30C45; Secondary 30C50.

Keywords and phrases: coefficient estimates, bi-Bazilevic functions, Mittag-Leffler, Chebyshev polynomials.

${ }^{*}$ Corresponding author 
Familiar subclasses of starlike and convex functions of order $\alpha(0 \leq \alpha<1)$ for which the quantity written respectively as $\operatorname{Re}\left(z f^{\prime}(z) / f(z)\right)>\alpha$ and $\operatorname{Re}\left(1+z f^{\prime \prime}(z) / f^{\prime}(z)\right)>\alpha$ and the two functions are denoted as $S^{*}(\alpha)$ and $C(\alpha)$ respectively. For a constant $\theta \in\left(-\frac{\pi}{2}, \frac{\pi}{2}\right)$, a function $f$ is univalent in $U$ and satisfies the function $\operatorname{Re}\left(e^{i \theta} \frac{z f^{\prime}(z)}{f(z)}\right) \geq \alpha$, denote the class by $T S^{*}$.

A function $f$ in $H$ has an inverse $f^{-1}$ defined as

$$
\begin{gathered}
f^{-1}(f(z))=z, \quad(z \in U) \\
f\left(f^{-1}(\omega)\right)=\omega, \quad\left(|\omega|<r_{o}(f), \quad r_{o}(f) \geq \frac{1}{4}\right)
\end{gathered}
$$

hence, the inverse function can be written as

$$
f^{-1}(\omega)=\omega-a_{2} \omega^{2}+\left(2 a_{2}^{2}-a_{3}\right) \omega^{3}-\left(5 a_{2}^{3}-5 a_{2} a_{3}+a_{4}\right) \omega^{4}+\ldots
$$

A function $f \in S$ which is starlike is bi-univalent in $U$ if both $f$ and $f^{-1}$ are both univalent in $U$. Let the class of all bi-univalent functions $f$ in $U$ be given by (1) denoted by $\sum$.

Lewin [12] introduced the concept of bi-univalent analytic functions in 1967 and showed that second coefficient $\left|a_{2}\right|<1.51$. Many authors like Netanyahu [15], Brannan and Clunie [8], Murugurusundaramoothy [14], Awolere and Ibrahim-Tiamiyu [6], Amol and Uday [5] have worked on bi-univalent analytic functions with interesting results. In recent time, several researchers have studied families of analytic functions involving special functions such as Sigmoid function, error function, Bessel function defined in $A$ to find different conditions such that members of such functions have certain geometric properties like univalency, starlikeness and convexity, see $[5,6,9,10,11,16]$. It was observed in $[3,4]$ that Mittag-Leffler function denoted by $E_{\lambda, \beta}$ does not belong to the family of $A$. Originally the Mittag-Leffler function is given as

$$
E_{\lambda}(z)=\sum_{k=2}^{\infty} \frac{z^{k}}{\Gamma(\lambda n+1)}, \quad(z \in C, R(\lambda)>0)
$$

which was introduced by Mittag-Leffler [13]. 
A more general form of (3) denoted by $E_{\lambda, \beta}(z)$ is defined as

$$
E_{\lambda, \beta}(z)=\sum_{k=2}^{\infty} \frac{z^{k}}{\Gamma(\lambda n+\beta)}, \quad(z, \lambda, \beta \in C, R(\lambda)>0) .
$$

The function in (3) arises naturally in the solution of fractional order differential and integral equations, most especially in the study of fractional generalization of kinetic equation, random walks levy flights, super diffusive transport and also in the study of complex systems. These polynomials interpolate between a purely exponential law and power law like behaviour of phenomena governed by ordinary kinetic equations and their fractional counterparts [3, 4].

By convolution and normalization, we obtain from (1) and (4)

$$
E_{\lambda, \beta} f(z)=z+\sum_{k=2}^{\infty} \frac{\Gamma(\beta)}{\Gamma(\lambda(k-1)+\beta)} a_{k} z^{k} .
$$

The Chebyshev polynomials are a sequence of orthogonal polynomials which are practically related to De Moivre's formula which play an important role in Numerical Analysis. Recently, the classical Chebyshev polynomials of degree $n$ of the first and second kinds have generated a great deal of interest because of the major roles played in Applied Mathematics and Approximation Theory. Chebyshev polynomials have been studied by several authors as in $[1,2,7,10,11$.

Lemma 1 (See 9). If $\omega(z)=b_{1} z+b_{2} z^{2}+b_{3} z^{3}+\ldots, b_{1} \neq 0$ is analytic and satisfies $|\omega(z)|<1$ on the unit disk $U$, then for each $0<r<1$, $\left|\omega^{\prime}(0)\right|<1$ and $\left|\omega\left(r e^{i \theta}\right)\right|<1$ unless $\omega(z)=e^{i \theta} z$ for some real number $\theta$.

Definition 1 (See 16). The Chebyshev polynomials of the first kind are defined by the following three terms recurrence relation

$$
\begin{array}{r}
T_{0}(t)=1 \\
T_{1}(t)=t \\
T_{n+1}(t)=2 t T_{n}(t)-T_{n-1}(t) .
\end{array}
$$

The first few terms of the Chebyshev polynomials of the first kind are

$$
T_{2}(t)=2 t^{2}-1, \quad T_{3}(t)=4 t^{3}-3 t, \quad T_{4}(t)=8 t^{4}-8 t^{2}+1, \ldots
$$


The generating function for Chebyshev polynomials of the first kind, $T_{n}(t)$ is given by

$$
F(z, t)=\frac{1-z t}{1-2 t z+z^{2}}=\sum_{n=0}^{\infty} T_{n}(t) z^{n}, \quad(z \in U)
$$

Definition 2 (See 16). The Chebyshev polynomials of the second kind are defined by the following three terms recurrence relation

$$
\begin{array}{r}
U_{0}(t)=1 \\
U_{1}(t)=2 t \\
U_{n+1}(t)=2 t U_{n}(t)-U_{n-1}(t) .
\end{array}
$$

The first few terms of the Chebyshev polynomials of the second kind, $U_{n}(t)$ is given by

$$
U_{2}(t)=4 t^{2}-1, \quad U_{3}(t)=8 t^{3}-4 t, \quad U_{4}(t)=16 t^{4}-12 t^{2}+1, \ldots
$$

The generating function for Chebyshev polynomials of the second kind, $U_{n}(t)$ is given by

$$
H(z, t)=\frac{1}{1-2 t z+z^{2}}=\sum_{n=0}^{\infty} U_{n}(t) z^{n}, \quad(z \in U) .
$$

The Chebyshev polynomial of first and second kinds are connected by the following relations

$$
\frac{d T_{n}(t)}{d t}=n U_{n-1}(t) ; \quad T_{n}(t)=U_{n}(t)-t T_{n-1}(t) ; \quad 2 T_{n}(t)=U_{n}(t)-U_{n-2}(t) .
$$

Given two functions $f, g \in A$. The function $f(z)$ is said to be subordinate to $g(z)$ in $U$, written $f(z) \prec g(z)$, if there exists a Schwarz function $\omega(z)$, analytic in $U$, with $\omega(0)=0$ and $|\omega(z)|<1$ for all $z \in U$ such that $f(z)=g(\omega(z))$.

Definition 3. A function $f(z) \in \sum$ given by (1) is said to be in the class $T_{\theta}^{\alpha}(\lambda, \beta, t)$ if the following conditions are satisfied

$$
e^{i \theta}\left(\frac{z^{1-\alpha}\left(E_{\lambda, \beta} f(z)\right)^{\prime}}{\left(E_{\lambda, \beta} f(z)\right)^{1-\alpha}}\right) \prec H(z, t) \cos \theta+i \sin \theta
$$


and

$$
e^{i \theta}\left(\frac{\omega^{1-\alpha}\left(E_{\lambda, \beta} g(\omega)\right)^{\prime}}{\left(E_{\lambda, \beta} g(\omega)\right)^{1-\alpha}}\right) \prec H(\omega, t) \cos \theta+i \sin \theta
$$

where $z, \omega \in U, \alpha \geq 0$ and the function $g \equiv f^{-1}$.

\section{Main Result}

Theorem 1. Let $f \in T_{\theta}^{\alpha}(\lambda, \beta, t)$, since $\beta, \lambda \in C, A_{k}$ is complex number. Then

$$
\begin{aligned}
\left|a_{2}\right| \leq & \frac{2 t \sqrt{2 t} \cos \theta}{\sqrt{\left|2 t^{2}\left[\left(\alpha^{2}+\alpha-2\right) A_{2}^{2}+2(\alpha+2) A_{3}\right] \cos \theta-2\left(4 t^{2}-1\right) e^{i \theta}\left(\alpha^{2}+2 \alpha+1\right) A_{2}^{2}\right|}} \\
\left|a_{3}\right| \leq & \left(\frac{2 t \cos \theta}{(\alpha+1)^{2} A_{2}}\right)^{2}+\frac{2 t \cos \theta}{(\alpha+2) A_{3}} \\
\left|a_{4}\right| \leq & \frac{10 t^{2} \cos ^{2} \theta}{\left(\alpha^{2}+3 \alpha+2\right) A_{2} A_{3}}+\frac{20 t^{3} \cos ^{3} \theta}{\left(\alpha^{3}+3 \alpha^{2}+3 \alpha+1\right) A_{2}^{3}} \\
& -\frac{4 M t^{3} \cos ^{3} \theta}{\left(\alpha^{3}+3 \alpha^{2}+3 \alpha+1\right)(\alpha+3) A_{2}^{3} A_{4}}+\frac{2\left(4 t^{3}+4 t^{2}-t-1\right) \cos \theta}{(\alpha+3) A_{4}}
\end{aligned}
$$

where $M=5(\alpha+3) A_{4}+2(\alpha-1)(\alpha+3) A_{2} A_{3}+\frac{(\alpha-1)(\alpha-2)(\alpha+3) A_{2}^{3}}{3}$ and $A_{k}=$ $\frac{\Gamma(\beta)}{\Gamma(\lambda(k-1)+\beta)}, k \geq 2$

Proof. From Definition 3

$$
e^{i \theta}\left(\frac{z^{1-\alpha}\left(E_{\lambda, \beta} f(z)\right)^{\prime}}{\left(E_{\lambda, \beta} f(z)\right)^{1-\alpha}}\right) \prec H(z, t) \cos \theta+i \sin \theta
$$

and

$$
e^{i \theta}\left(\frac{\omega^{1-\alpha}\left(E_{\lambda, \beta} g(\omega)\right)^{\prime}}{\left(E_{\lambda, \beta} g(\omega)\right)^{1-\alpha}}\right) \prec H(\omega, t) \cos \theta+i \sin \theta
$$

where $H(z, t)$ and $H(\omega, t)$ are Chebyshev polynomials with the following power series

$$
H(z, t)=1+c_{1} u_{1}(t) z+\left(c_{2} u_{1}(t)+c_{1}^{2} u_{2}(t)\right) z^{2}+\ldots \quad(z \in U)
$$




$$
H(\omega, t)=1+d_{1} u_{1}(t) \omega+\left(d_{2} u_{1}(t)+d_{1}^{2} u_{2}(t)\right) \omega^{2}+\ldots \quad(\omega \in U) .
$$

For some analytic $\varphi, \psi$ such that $\varphi(0)=\psi(0)=0$ and $|\varphi(z)|=\left|c_{1} z+c_{2} z^{2}+\ldots\right|<$ $1,|\psi(\omega)|=\left|d_{1} \omega+d_{2} \omega^{2}+\ldots\right|<1$, for all $z, \omega \in U$. It is well known that $\left|c_{k}\right| \leq$ $1,\left|d_{k}\right| \leq 1$; for every $k \in N$.

Now, by (11) and (12), we have

$$
\begin{gathered}
(\alpha+1) A_{2} a_{2}=c_{1} u_{1}(t) e^{-i \theta} \cos \theta \\
\frac{(\alpha-1)(\alpha+2)}{2} A_{2}^{2} a_{2}^{2}+(\alpha+2) A_{3} a_{3}=c_{2} u_{1}(t) e^{-i \theta} \cos \theta+c_{1}^{2} u_{2}(t) e^{-i \theta} \cos \theta \\
(\alpha+3) A_{4} a_{4}+(\alpha-1)(\alpha+3) A_{2} A_{3} a_{2} a_{3}+\frac{(\alpha-1)(\alpha-2)(\alpha+3)}{6} A_{2}^{3} a_{2}^{3} \\
=c_{3} u_{1}(t) e^{-i \theta} \cos \theta+2 c_{1} c_{2} u_{2}(t) e^{-i \theta} \cos \theta+c_{1}^{3} u_{3}(t) e^{-i \theta} \cos \theta-(\alpha+1) A_{2} a_{2} \\
=d_{1} u_{1}(t) e^{-i \theta} \cos \theta \\
{\left[2(\alpha+2) A_{3}+\frac{(\alpha-1)(\alpha+2)}{2} A_{2}^{2}\right] a_{2}^{2}-(\alpha+2) A_{3} a_{3}} \\
=d_{2} u_{1}(t) e^{-i \theta} \cos \theta+d_{1}^{2} u_{2}(t) e^{-i \theta} \cos \theta
\end{gathered}
$$

and

$$
\begin{aligned}
& {\left[5(\alpha+3) A_{4}+(\alpha-1)(\alpha+3) A_{2} A_{3}\right] a_{2} a_{3} } \\
& -\left[5(\alpha+3) A_{4}+2(\alpha-1)(\alpha+3) A_{2} A_{3}+\frac{(\alpha-1)(\alpha-2)(\alpha+3)}{6} A_{2}^{3}\right] a_{2}^{3} \\
& -(\alpha+3) A_{4} a_{4} \\
= & d_{3} u_{1}(t) e^{-i \theta} \cos \theta+2 d_{1} d_{2} u_{2}(t) e^{-i \theta} \cos \theta+d_{1}^{3} u_{3}(t) e^{-i \theta} \cos \theta
\end{aligned}
$$

From (13) and (15), we have

$$
a_{2}=\frac{c_{1} u_{1}(t) e^{-i \theta} \cos \theta}{(\alpha+1) A_{2}}=-\frac{d_{1} u_{1}(t) e^{-i \theta} \cos \theta}{(\alpha+1) A_{2}}
$$

which implies that

$$
c_{1}=-d_{1}
$$


and

$$
2 e^{2 i \theta}(\alpha+1)^{2} A_{2}^{2} a_{2}^{2}=\left(c_{1}^{2}+d_{1}^{2}\right) \cos ^{2} \theta+u_{1}^{2}(t) .
$$

By equations (14) and (16), we have

$$
\frac{e^{i \theta}}{\cos \theta}\left[(\alpha-1)(\alpha+2) A_{2}^{2}+2(\alpha+2) A_{3}\right] a_{2}^{2}=\left(c_{2}+d_{2}\right) u_{1}(t)+\left(c_{1}^{2}+d_{1}^{2}\right) u_{2}(t)
$$

Now, by (20) and (21)

$$
a_{2}^{2}=\frac{u_{1}^{3}(t)\left(c_{2}+d_{2}\right) e^{-i \theta} \cos ^{2} \theta}{u_{1}^{2}(t)\left[\left(\alpha^{2}+\alpha-2\right) A_{2}^{2}+2(\alpha+2) A_{3}\right] \cos \theta-2 e^{i \theta} u_{2}(t)\left(\alpha^{2}+2 \alpha+1\right) A_{2}^{2}} .
$$

Since by definition, $u_{1}(t)=2 t, u_{2}(t)=4 t^{2}-1$ and $u_{3}(t)=8 t^{3}-4 t$.

Applying Lemma 1 for the coefficients $c_{2}$ and $d_{2}$, we immediately have

$$
\left|a_{2}\right| \leq \frac{2 t \sqrt{2 t} \cos \theta}{\sqrt{\left|2 t^{2}\left[\left(\alpha^{2}+\alpha-2\right) A_{2}^{2}+2(\alpha+2) A_{3}\right] \cos \theta-2\left(4 t^{2}-1\right) e^{i \theta}\left(\alpha^{2}+2 \alpha+1\right) A_{2}^{2}\right|}} .
$$

Next, to find the bound on $\left|a_{3}\right|$, we subtract (16) from (14) to get

$$
\frac{e^{i \theta}}{\cos \theta}\left[2(\alpha+2) A_{3} a_{3}-2(\alpha+2) A_{3} a_{2}^{2}\right]=u_{1}(t)\left(c_{2}-d_{2}\right)+u_{2}(t)\left(c_{1}^{2}-d_{1}^{2}\right) .
$$

Thus, it follows from (19), (20) and (24) that

$$
a_{3}=\frac{u_{1}^{2}(t)\left(c_{1}^{2}+d_{1}^{2}\right) e^{-2 i \theta} \cos ^{2} \theta}{2(\alpha+1)^{2} A_{2}^{2}}+\frac{u_{1}(t)\left(c_{2}-d_{2}\right) e^{-i \theta} \cos \theta}{2(\alpha+2) A_{3}} .
$$

By Lemma 1 , the coefficients $c_{1}, d_{1}, c_{2}$ and $d_{2}$ yield

$$
\left|a_{3}\right| \leq\left(\frac{2 t \cos \theta}{(\alpha+1)^{2} A_{2}}\right)^{2}+\frac{2 t \cos \theta}{(\alpha+2) A_{3}} .
$$

Furthermore, by (13), (15), (17) and (25), we have

$$
\begin{aligned}
a_{4}= & \frac{5 u_{1}^{2}(t) c_{1}\left(c_{2}-d_{2}\right) e^{-2 i \theta} \cos ^{2} \theta}{4(\alpha+1)(\alpha+2) A_{2} A_{3}}+\frac{5 u_{1}^{3}(t) c_{1}\left(c_{1}^{2}+d_{1}^{2}\right) e^{-3 i \theta} \cos ^{3} \theta}{4(\alpha+1)^{3} A_{2}^{3}} \\
& -\frac{M u_{1}^{3}(t) c_{1}^{3} e^{-3 i \theta} \cos ^{3} \theta}{2(\alpha+1)^{3}(\alpha+3) A_{2}^{3} A_{4}}+\frac{2 u_{2}(t)\left(c_{1} c_{2}-d_{1} d_{2}\right) e^{-i \theta} \cos \theta}{2(\alpha+3) A_{4}} \\
& +\frac{u_{1}(t)\left(c_{3}-d_{3}\right) e^{-i \theta} \cos \theta}{2(\alpha+3) A_{4}}+\frac{u_{3}(t)\left(c_{1}^{3}-d_{1}^{3}\right) e^{-i \theta} \cos \theta}{2(\alpha+3) A_{4}} .
\end{aligned}
$$


On application of Lemma 1 for coefficients $c_{1}, d_{1}, c_{2}, d_{2}, c_{3}$ and $d_{3}$, then

$$
\begin{aligned}
\left|a_{4}\right| \leq & \frac{10 t^{2} \cos ^{2} \theta}{\left(\alpha^{2}+3 \alpha+2\right) A_{2} A_{3}}+\frac{20 t^{3} \cos ^{3} \theta}{\left(\alpha^{3}+3 \alpha^{2}+3 \alpha+1\right) A_{2}^{3}} \\
& -\frac{4 M t^{3} \cos ^{3} \theta}{\left(\alpha^{3}+3 \alpha^{2}+3 \alpha+1\right)(\alpha+3) A_{2}^{3} A_{4}}+\frac{2\left(4 t^{3}+4 t^{2}-t-1\right) \cos \theta}{(\alpha+3) A_{4}}
\end{aligned}
$$

where

$$
M=5(\alpha+3) A_{4}+2(\alpha-1)(\alpha+3) A_{2} A_{3}+\frac{(\alpha-1)(\alpha-2)(\alpha+3) A_{2}^{3}}{3}
$$

which yield the required result, hence the proof completes.

Corollary 1. Let $f \in T_{0}^{\alpha}(\lambda, \beta, t)$, since $\beta, \lambda \in C, A_{k}$ is complex number. Then

$$
\begin{aligned}
\left|a_{2}\right| \leq & \frac{2 t \sqrt{2 t}}{\sqrt{\left|2 t^{2}\left[\left(\alpha^{2}+\alpha-2\right) A_{2}^{2}+2(\alpha+2) A_{3}\right]-2\left(4 t^{2}-1\right)\left(\alpha^{2}+2 \alpha+1\right) A_{2}^{2}\right|}} \\
\left|a_{3}\right| \leq & \frac{2 t}{(\alpha+1)^{2} A_{2}}+\frac{2 t}{(\alpha+2) A_{3}} \\
\left|a_{4}\right| \leq & \frac{10 t^{2}}{\left(\alpha^{2}+3 \alpha+2\right) A_{2} A_{3}}+\frac{20 t^{3}}{\left(\alpha^{3}+3 \alpha^{2}+3 \alpha+1\right) A_{2}^{3}} \\
& -\frac{4 M t^{3}}{\left(\alpha^{3}+3 \alpha^{2}+3 \alpha+1\right)(\alpha+3) A_{2}^{3} A_{4}}+\frac{2\left(4 t^{3}+4 t^{2}-t-1\right)}{(\alpha+3) A_{4}} .
\end{aligned}
$$

Corollary 2. Let $f \in T_{0}^{0}(\lambda, \beta, t)$, since $\beta, \lambda \in C, A_{k}$ is complex number. Then

$$
\begin{aligned}
\left|a_{2}\right| & \leq \frac{t \sqrt{2 t}}{\sqrt{\left|2 A_{3}+\left(1-6 t^{2}\right) A_{2}^{2}\right|}} \\
\left|a_{3}\right| & \leq \frac{2 t}{A_{2}}+\frac{t}{A_{3}} \\
\left|a_{4}\right| & \leq \frac{5 t^{2}}{A_{2} A_{3}}+\frac{20 t^{3}}{A_{2}^{3}}-\frac{4 M t^{3}}{3 A_{2}^{3} A_{4}}+\frac{2\left(4 t^{3}+4 t^{2}-t-1\right)}{3 A_{4}} .
\end{aligned}
$$


Corollary 3. Let $f \in T_{\theta}^{\alpha}(\lambda, \beta, 1)$, since $\beta, \lambda \in C, A_{k}$ is complex number. Then

$$
\begin{aligned}
\left|a_{2}\right| \leq & \frac{\sqrt{2} \cos \theta}{\sqrt{\left|\cos \theta\left[\left(\alpha^{2}+\alpha-2\right) A_{2}^{2}+2(\alpha+2) A_{3}\right]-3 e^{i \theta}\left(\alpha^{2}+2 \alpha+1\right) A_{2}^{2}\right|}} \\
\left|a_{3}\right| \leq & \left(\frac{2 \cos \theta}{(\alpha+1)^{2} A_{2}}\right)^{2}+\frac{2 \cos \theta}{(\alpha+2) A_{3}} \\
\left|a_{4}\right| \leq & \frac{10 \cos ^{2} \theta}{\left(\alpha^{2}+3 \alpha+2\right) A_{2} A_{3}}+\frac{20 \cos ^{3} \theta}{\left(\alpha^{3}+3 \alpha^{2}+3 \alpha+1\right) A_{2}^{3}} \\
& -\frac{4 M \cos ^{3} \theta}{\left(\alpha^{3}+3 \alpha^{2}+3 \alpha+1\right)(\alpha+3) A_{2}^{3} A_{4}}+\frac{12 \cos \theta}{(\alpha+3) A_{4}} .
\end{aligned}
$$

Corollary 4. Let $f \in T_{0}^{\alpha}(\lambda, \beta, 1)$, since $\beta, \lambda \in C, A_{k}$ is complex number. Then

$$
\begin{aligned}
\left|a_{2}\right| \leq & \frac{\sqrt{2}}{\sqrt{\left|\left[\left(\alpha^{2}+\alpha-2\right) A_{2}^{2}+2(\alpha+2) A_{3}\right]-3\left(\alpha^{2}+2 \alpha+1\right) A_{2}^{2}\right|}} \\
\left|a_{3}\right| \leq & \left(\frac{2}{(\alpha+1)^{2} A_{2}}\right)^{2}+\frac{2}{(\alpha+2) A_{3}} \\
\left|a_{4}\right| \leq & \frac{10}{\left(\alpha^{2}+3 \alpha+2\right) A_{2} A_{3}}+\frac{20}{\left(\alpha^{3}+3 \alpha^{2}+3 \alpha+1\right) A_{2}^{3}} \\
& -\frac{4 M}{\left(\alpha^{3}+3 \alpha^{2}+3 \alpha+1\right)(\alpha+3) A_{2}^{3} A_{4}}+\frac{12}{(\alpha+3) A_{4}} .
\end{aligned}
$$

Corollary 5. Let $f \in T_{0}^{0}(\lambda, \beta, 1)$, since $\beta, \lambda \in C, A_{k}$ is complex number. Then

$$
\begin{aligned}
\left|a_{2}\right| & \leq \frac{\sqrt{2}}{\sqrt{\left|4 A_{3}-5 A_{2}^{2}\right|}} \\
\left|a_{3}\right| & \leq \frac{4}{A_{2}^{2}}+\frac{1}{A_{3}} \\
\left|a_{4}\right| & \leq \frac{5}{A_{2} A_{3}}+\frac{20}{A_{2}^{3}}-\frac{4 M}{3 A_{2}^{3} A_{4}}+\frac{4}{A_{4}} .
\end{aligned}
$$

Theorem 2. Let $f \in T_{\theta}^{\alpha}(\lambda, \beta, t)$ and $\varphi \in R$, since $\beta, \lambda \in C, A_{k}$ is complex 
number. Then

$$
\leq\left\{\begin{array}{cc}
\left|a_{3}-\varphi a_{2}^{2}\right| & |\varphi-1| \leq M \\
\frac{2 t \cos \theta}{(\alpha+2) A_{3}}, & |\varphi-1| \geq M \\
\frac{8|1-\varphi| t^{3} \cos ^{2} \theta}{2 t^{2}\left[\left(\alpha^{2}+\alpha-2\right) A_{2}^{2}+2(\alpha+2) A_{3}\right] \cos \theta-\left(4 t^{2}-1\right) e^{i \theta}\left(\alpha^{2}+2 \alpha+1\right) A_{2}^{2} \mid}, & |\varphi-1| \geq
\end{array}\right.
$$

where

$$
M=\frac{\left|2 t^{2}\left[\left(\alpha^{2}+\alpha-2\right) A_{2}^{2}+2(\alpha+2) A_{3}\right] \cos \theta-\left(4 t^{2}-1\right) e^{i \theta}\left(\alpha^{2}+2 \alpha+1\right) A_{2}^{2}\right|}{4 t^{2} \cos \theta(\alpha+2) A_{3}},
$$

Proof.

$$
\begin{aligned}
a_{3}-\varphi a_{2}^{2}= & \frac{(1-\varphi) u_{1}^{3}(t)\left(c_{2}+d_{2}\right) e^{-i \theta} \cos ^{2} \theta}{u_{1}^{2}(t)\left[\left(\alpha^{2}+\alpha-2\right) A_{2}^{2}+2(\alpha+2) A_{3}\right] \cos \theta-2 e^{i \theta} u_{2}(t)\left(\alpha^{2}+2 \alpha+1\right) A_{2}^{2}} \\
& +\frac{u_{1}(t)\left(c_{2}-d_{2}\right) e^{-i \theta} \cos \theta}{2(\alpha+2) A_{3}} \\
= & u_{1}(t) e^{-i \theta} \cos \theta\left[\left(h(\varphi)+\frac{1}{2(\alpha+2) A_{3}}\right) c_{2}+\left(h(\varphi)-\frac{1}{2(\alpha+2) A_{3}}\right) d_{2}\right]
\end{aligned}
$$

where

$$
h(\varphi)=\frac{(1-\varphi) u_{1}^{2}(t) \cos \theta}{u_{1}^{2}(t)\left[\left(\alpha^{2}+\alpha-2\right) A_{2}^{2}+2(\alpha+2) A_{3}\right] \cos \theta-2 e^{i \theta} u_{2}(t)\left(\alpha^{2}+2 \alpha+1\right) A_{2}^{2}}
$$

since $u_{1}(t)=2 t, \quad u_{2}(t)=4 t^{2}-1$ and $u_{3}(t)=8 t^{3}-4 t$, then we have

$$
\left|a_{3}-\varphi a_{2}^{2}\right| \leq\left\{\begin{array}{cc}
\frac{2 t \cos \theta}{(\alpha+2) A_{3}}, & 0 \leq|h(\varphi)| \leq \frac{1}{2(\alpha+2) A_{3}} \\
4 t|h(\varphi)| \cos \theta, & |h(\varphi)| \geq \frac{1}{2(\alpha+2) A_{3}}
\end{array}\right.
$$




\section{References}

[1] S. Altinkaya and S. Yalcin, Initial coefficient bounds for a general class of biunivalent functions, International Journal of Analysis 20 (2014), Article ID 867871. https://doi.org/10.1155/2014/867871

[2] S. Altinkaya and S. Yalcin, Coefficient bounds for a subclass of bi-univalent functions, TWMS Journal of Pure and Applied Mathematics 6(2) (2015), 180-185.

[3] S. Altinkaya and S. Yalcin, Coefficient estimates for two new subclasses of biunivalent functions with respect to symmetric points, Journal of Function Spaces 2015 (2015), Article ID 145242. https://doi.org/10.1155/2015/145242

[4] S. Altinkaya and S. Yalcin, On the Chebyshev polynomial coefficient problem of some subclasses of bi-univalent functions, Gulf Journal of Mathematics 5 (2017), 34-40.

[5] B. P. Amol and H. N. Uday, Estimate on coefficients of certain subclasses of bi-univalent functions associated with Hohlov operator, Palestine Journal of Mathematics 7(2) (2018), 487-497.

[6] I. T. Awolere and S. Ibrahim-Tiamiyu, New subclasses of bi-univalent pseudo starlike function using Alhindi-Darus generalized hypergeometric function, Journal of the Nigerian Association of Mathematical Physics 40 (2017), 65-72.

[7] D. A. Brannan, J. Clunie and W. E. Kirwan, Coefficient estimates for a class of star-like functions, Canadian Journal of Mathematics 22 (1970), 476-485. https://doi.org/10.4153/CJM-1970-055-8

[8] D. A. Brannan and J. Clunie (Eds.), Aspects of Contemporary Complex Analysis, Proceedings of the NATO Advanced Study Institute (University of Durham, Durham; July, 1979), Academic Press, New York and London, 1980.

[9] P. L. Duren, Univalent functions, Grundlehren der Math. Wiss. 259, Springer-Verlag, New York, Berlin, Heidelberg, Tokyo, 1983.

[10] M. Fekete and G. Szego, Eine bemerkung uber ungerade schlichte funktionen, J. London Math. Soc. 8 (1933), 85-89. https://doi.org/10.1112/jlms/s1-8.2.85

[11] B. A. Frasin and M. K. Aouf, New subclasses of bi-univalent functions, Appl. Math. Lett. 24 (2011), 1569-1573. https://doi.org/10.1016/j.aml.2011.03.048 
[12] M. Lewin, On the coefficient problem for bi-univalent functions, Proc. Amer. Math. Soc. 18 (1967), 63-68. https://doi.org/10.1090/S0002-9939-1967-0206255-1

[13] G. Mittag-Leffler, Sur la nouvelle fonction $E_{\alpha}(x)$, C. R. Acad. Sci. Paris 137 (1903), 554-558.

[14] G. Murugusundaramoorthy, Coefficient estimates of bi-Bazileviuc function of complex order based on quasi subordination involving Srivastava-Attiya operator, 2016. arXiv:1601.0229vl [math.cv]

[15] E. Netanyahu, The minimal distance of the image boundary from origin and second coefficient of a univalent function in $|z|<1$, Arch. Ration Mech. Anal. 32 (1969), 100-112. https://doi.org/10.1007/BF00247676

[16] F. Yousef, B. A. Frasin and Tariq Al-Hawary, Fekete-Szego inequality for analytic and bi-univalent functions subordinate to Chebyshev polynomials, 2018. arXiv:1801.09531 [math.CV]

This is an open access article distributed under the terms of the Creative Commons Attribution License (http://creativecommons.org/licenses/by/4.0/), which permits unrestricted, use, distribution and reproduction in any medium, or format for any purpose, even commercially provided the work is properly cited. 